\title{
A DFT study of thermal and structural properties of 2D layers (Short title: Two-Dimensional materials)
}

\author{
Abdul Majid ${ }^{1}$, Hajra Kanwal ${ }^{1}$, Salah Khan ${ }^{2}$, and Ashfaq Ahmad ${ }^{2}$ \\ ${ }^{1}$ University of Gujrat \\ ${ }^{2}$ King Saud University
}

November 21, 2020

\begin{abstract}
Two-dimensional (2D) materials have exhibited exceptional properties which meet the demands of future applications. These materials appeared after discovery of graphene in 2004 offered such device grade characteristics at nanoscale which did not appear on bulk scale. The research turned to search alternate 2D materials when drawbacks of graphene became surfaced. Despite significant successes and unprecedented efforts which consequent upon several beyond-graphene 2D materials, the complete potentials of such materials are still unexplored which may restrict their usage in devices. This work was carried out with motivation to investigate the thermal stability of several 2D-mono-layered materials including graphene, Borophene, Aluminene, Germanene, BN, SiC and MoS2 based on classical Molecular Dynamics Simulations. Prior to the implementation of the conditions for thermal calculations, the structures were optimized using Geometry-Optimization method. It appeared that all the structural parameters which includes lattice-constant, bond-length and dihedral angles were precisely determined. On the contrary, it was found that several materials beyond graphene can resist up-to certain temperature ranges, depicting the material dependent thermal stability. The radial distribution function (RDF) was calculated which pointed towards thermal broadening, bond breakage and bond formation for the slabs. The RDF-peaks were found to characterize the probability of finding any particle in the nearest neighbors which extend the phenomenon of thermal stability. Thermal stability was compared by plotting the temperature and energy curves from which, the phase transition temperature and heat capacity was determined for the slabs including graphene as benchmark. The phase transition temperatures are found as $4510 \mathrm{~K}$, $2273 \mathrm{~K}$, $933 \mathrm{~K}, 1670 \mathrm{~K}, 3246 \mathrm{~K}, 4050 \mathrm{~K}$, and $1460 \mathrm{~K}$ for graphene, Borophene, Aluminene, Germanene, BN, SiC and MoS2 respectively. Besides the analysis of temperature-energy variations, the thermal broadening is also determined and discussed to examine the thermal-stability for usage of the materials in high temperature applications.
\end{abstract}

\section{Hosted file}

Hajra_paper_19.11.2020.pdf available at https://authorea.com/users/291373/articles/494247-adft-study-of-thermal-and-structural-properties-of-2d-layers-short-title-two-dimensionalmaterials 